\title{
Development of an on-line cell-based bioactivity screening method by coupling liquid chromatography to flow cytometry with parallel mass spectrometry
}

\author{
Reka A. Otvos $^{\dagger \ddagger}$, Pim van Nierop ${ }^{\ddagger}$, Wilfried M.A. Niessen ${ }^{\dagger \S}$, R. Manjunatha Kinill, Govert W. \\ Somsen ${ }^{\dagger}$, August B. Smit ${ }^{\ddagger}$ and Jeroen $\mathrm{Kool}^{\star}+$ \\ † Division of BioAnalytical Chemistry, Vrije Univesiteit Amsterdam, De Boelelaan 1083, 1081 HV Amsterdam, The \\ Netherlands \\ ‡ Department of Molecular and Cellular Neurobiology, Vrije Universiteit Amsterdam, De Boelelaan 1085, 1081 HV \\ Amsterdam, The Netherlands
}

$\S$ hyphen MassSpec, Herenweg 95, 2361 EK Warmond, The Netherlands

॥ Department of Biological Sciences, National University of Singapore, 14 Science Drive 4, 117543, Singapore

*j.kool@vu.nl

\begin{abstract}
This study describes a new platform for the fast and efficient functional screening for bioactives, or bioactive compounds, in complex natural mixtures using a cell-based assay. For this, the platform combines reversed-phase liquid chromatography (LC) with on-line flow cytometry (FC) and mass spectrometry (MS). As a model (an example or proof-ofconcept study) we have used a functional calcium-flux assay in human neuroblastoma $\mathrm{SH}_{-} \mathrm{SY}_{5} \mathrm{Y}$ cells stably overexpressing the alpha-7 nicotinic acetylcholine receptor $(\alpha 7-n A C h R)$, a potential therapeutic target for central nervous system (CNS) related diseases. We have designed the coupled LC-FC system employing the neuroblastoma cells followed by analytical and pharmacological evaluation of the hyphenated setup in agonist and mixed antagonist-agonist assay modes. Using standard receptor ligands we have validated pharmacological responses and standardized good assay quality parameters. The applicability of the screening system was evaluated by analysis of various types of natural samples, such as a tobacco plant extract (in agonist assay mode) and snake venoms (in mixed antagonist-agonist assay mode). The bioactivity responses were correlated directly to the respective accurate masses of the compounds. Using simultaneous functional agonist and antagonist responses nicotine and known neurotoxins were detected from tobacco extract and snake venoms, respectively. Thus, the developed analytical screening technique represents a new tool for rapid measurement of functional cell-based responses and parallel separation and identification of compounds in complex mixtures targeting the $\alpha_{7}-\mathrm{nAChR}$. It is anticipated that other fast-response cell-based assays (e.g. other ion flux assays) can be incorporated in this analytical setup.
\end{abstract}

\section{Supporting information table of contents:}

Process of converting FC raw data into chromatographic format.

Calculation of final assay concentrations.

Calculation of assay statistical parameters.

The R script used for the generation of data points for converted FC bioassay chromatograms.

Figure S-1. Chemical structure of standard compounds.

Figure S-2. Hardware modifications of the FC instrument.

Figure S-3. Effect of using different gating during the FC data analysis.

Figure S-4. The effect of organic solvents and salts on SH-SY5Y cells in plate-reader format.

Figure S-5. The effect of organic solvents and salts on SH-SY5Y cells in on-line LC-FC assay format.

Figure S-6. Determination of cell viability by propidium iodide (PI) staining.

Figure S-7. Intraday repeatability and interday reproducibility in agonist mode. 
Figure S-8. Intraday repeatability and interday reproducibility in mixed antagonist-agonist mode.

Figure S-9. Proof-of-principle experiment showing the performance of the antagonist setup.

Figure S-10: Simultaneous agonist-antagonist measurement in antagonist mode.

Figure S-11. On-line cell-based bioactivity screening of Dendroapsis polylepis snake venom.

\section{Process of converting FC raw data into chromatographic format}

After gating all points representing living and functional cells (see SI Figure S-2), the raw data from the MXP software was exported into .csv files using the Export compensated data function of the Kaluza software (Beckman Coulter). Subsequently, the 1-s average of the FL1 channel was calculated using an in-house written R Studio software (Boston, MA, United States) algorithm (Supporting information). Since the MXP software displays and stores events in time as a 1024 channel format, the following calculation was used to transform this format into measured events per second for each channel:

timesec $=$ time1024* (timeacq/timelast channel); where timesec is the real time displayed in seconds, time1024 is the time displayed in the exported .csf file, timeacq is the real acquisition time of the measurement, and timelast channel is the time of the last channel in the exported .csf file. The final converted results were displayed in time against the mean of relative fluorescence in FL1 channel per second using GraphPad Prism 5 software (GraphPad Software Inc., San Diego, CA).

\section{Calculation of final assay concentrations}

The final concentration in the assay was calculated as described by Falck et al. $2010^{1}$. In short, the final concentration is determined by the mixing dilution $\left(\mathrm{D}_{\mathrm{M}}\right)$ and the chromatographic dilution $\left(\mathrm{D}_{\mathrm{C}}\right)$.

$$
D_{M}=\frac{u_{F}}{u_{E}}
$$

where $u_{F}$ is the final flow rate in the bioassay detector and $u_{E}$ is the flow rate of the LC eluent mixing with the other components of the assay.

$$
D_{c}=\frac{F W H M}{2} \cdot \sqrt{\frac{\pi}{\ln 2}} \cdot \frac{u_{C}}{V_{i}}
$$

where FWHM is the full width at half maximum, $\mathrm{u}_{\mathrm{C}}$ is the LC flowrate and $\mathrm{V}_{\mathrm{i}}$ is the injection volume.

$$
c_{F}=\frac{c_{i}}{D_{M} \cdot D_{C}} \quad(3)
$$


Where $c_{F}$ is the final assay concentration, $c_{i}$ is the injected concentration.

\section{Calculation of assay statistical parameters}

For determination of the assay quality, a modified equation of $Z$ '-factor, the $Z$ ' chrom (equation 4) value was used, which is well suited for describing on-line assays ${ }^{1}$. The reasons for the suitability of $Z$ ' chrom instead of Z'-factor for describing assay quality of chromatography-based on-line assays is described in detail by Falck et al. ${ }^{1}$.

$$
\mathrm{Z}_{\text {chrom }}^{\prime}=1-\frac{\left(3 \mathrm{SD}_{\mathrm{H}}+3 \cdot \text { noise }\right)}{\left|\mu_{\mathrm{H}}\right|}
$$

Where $\mathrm{SD}_{\mathrm{H}}$ is the standard deviation of the peaks obtained from full activation signal, $\mu_{\mathrm{H}}$ is the average of the peak height of full activation (which is equivalent to the assay window, or $\mu_{\mathrm{c}}-\mu_{\mathrm{s}}$, the difference of the average of full activation and no activation. Noise refers to the chromatographic baseline deviation.

Dynamic range is calculated as the difference between the average of peak height of maximum signal $\left(\mu_{\mathrm{H}}\right)$ and the average of noise.

The signal to noise ratio $(\mathrm{S} / \mathrm{N})$ is defined according to Zhang et al. ${ }^{2}$ as equation 5.

$S / N=\frac{\text { mean signal-mean background }}{\text { standard deviation of background }}$

The signal to background (S/B) is defined according to Zhang et al. ${ }^{2}$ as equation 6 .

$S / B=\frac{\text { mean signal }}{\text { mean background }}$

The $R$ script used for the generation of data points for converted FC bioassay chromatograms

convert.data $<$-function(filename,directory) \{

$\operatorname{library}(\mathrm{plyr})$

data $=$ read.csv(paste(directory,filename,sep=""))

acc.time $=$ as.numeric $($ gsub("\\.act| $\mid \backslash . c s v ", " "$, str_extract(string=filename,pattern = $\left.\left.\left." \backslash \backslash . \operatorname{act} \backslash \backslash d+\backslash \backslash . \operatorname{csv}^{\prime}\right)\right)\right)$

lastbucket $=$ data\$TIME[length $($ data\$TIME $)]$

scale.factor $=$ acc.time/lastbucket

data\$TIME. $\sec =$ data\$TIME $*$ scale.factor 
summary.data $=$ ddply $($ data,..$($ TIME.sec),summarise,avg.FL1=mean(FL1))

new.filename = paste(directory,paste("converted",filename,sep = "_"),sep="")

dev.off()

write.table(summary.data,file = new.filename, sep = ",", col.names = NA, qmethod = "double") $\}$

file.directory = "U:/flow cytometer/" \# mid the trailing '/' it must be present here

files $=$ list.files(file.directory, pattern="*.csv",full.names=F)

for (file in files) \{convert.data(file,file.directory)\}

\section{References}

(1) Falck, D.; de Vlieger, J. S.; Niessen, W. M.; Kool, J.; Honing, M.; Giera, M.; Irth, H. Anal Bioanal Chem 2010, 398, 1771-1780.

(2) Zhang, J. H.; Chung, T. D.; Oldenburg, K. R. J Biomol Screen 1999, 4, 67-73. 
Figure S-1. Chemical structures of (a) nicotine, (b) PNU120596, (c) PNU282987, (d) MLA, (e) BTX.

a)

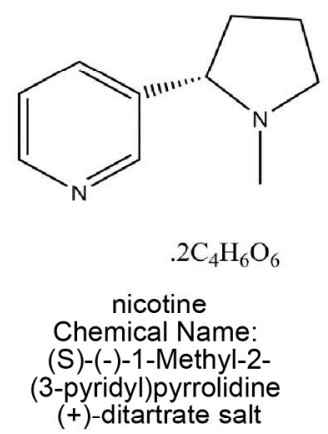

b)

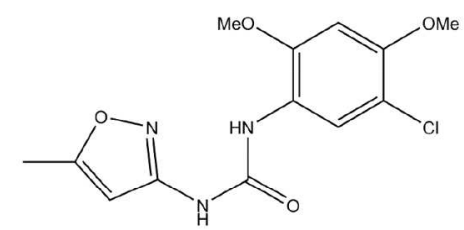

PNU120596

Chemical Name: N-(5-Chloro-2,4dimethoxyphenyl)-

N'-(5-methyl-3-isoxazolyl)- c)<smiles>O=C(N[C@H]1CN2CCC1CC2)c1ccc(Cl)cc1</smiles>

PNU282987

Chemical Name: N-(3R)-

1-Azabicyclo

[2.2.2]oct-3-yl-4-chlorobenzamide d)

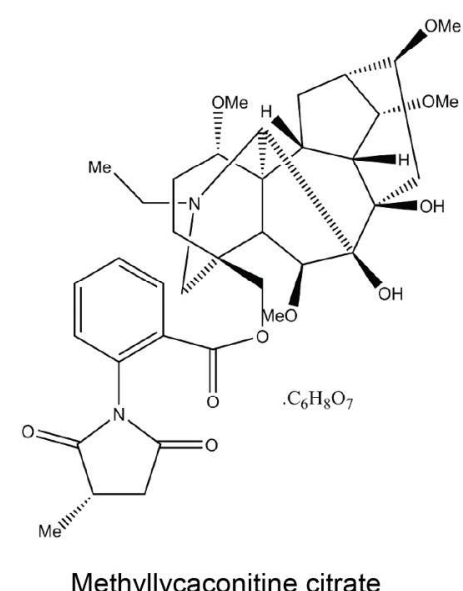

Chemical Name: [1 $\alpha, 4(S), 6 \beta, 14 \alpha, 16 \beta]-20-E$ thyl-

1,6,14,16-tetramethoxy-4-[[[2-(3-methyl-2,5-dioxo-

1-pyrrolidinyl)benzoyl]oxy]methyl]aconitane-7,8-diol e)

$$
\begin{aligned}
& \text { Ile-Val-Cys-His-Thr-Thr-Ala-Thr-Ser-Pro- } \\
& \text { lle-Ser-Ala-Val-Thr-Cys-Pro-Pro-Gly-Glu- } \\
& \text { Asn-Leu-Oys-Tyr-Arg-Lys-Met-Trp-Oys-Asp- } \\
& \text { Ala-Phe-Cys-Ser-Ser-Arg-Gly-Lys-Val-Val- } \\
& \text { Glu-Leu-Gly-Cys-Ala-Ala-Thr-Oys-Pro-Ser- } \\
& \text { Lys-Lys-Pro-Tyr-Glu-Glu-Val-Thr-Cys-C/s- } \\
& \text { Ser-Thr-Asp-Lys-oys-Asn-Pro-His-Pro-Lys- } \\
& \text { Gln-Arg-Pro-Gly } \\
& \text { a-Bungarotoxin }
\end{aligned}
$$


Figure S-2. Hardware modifications of the FC instrument. (A) The original sample flow in the FC instrument. The samples are placed in the sample rack (1) of the FC instrument. After injection by the sample probe (2), the sample is directed into the flow cell (4) through the sample pickup tubing (3). (B) Modified sample flow in the FC instrument with the continuous flow online FC bioassay. A dummy sample is placed in the sample rack (1) of the flow cytometer. The sample pickup tubing (3) is disconnected from the flow cell (4) and directed to a switch valve (5). During the start/end of the measurement the switch valve is open and the flow is directed to waste (6). During measurement time the switch valve is in stop position (7). The tubing of the continuous flow on-line bioassay (8) is directly connected to the flow cell of the FC instrument (4).

\section{A Original sample flow in FC}
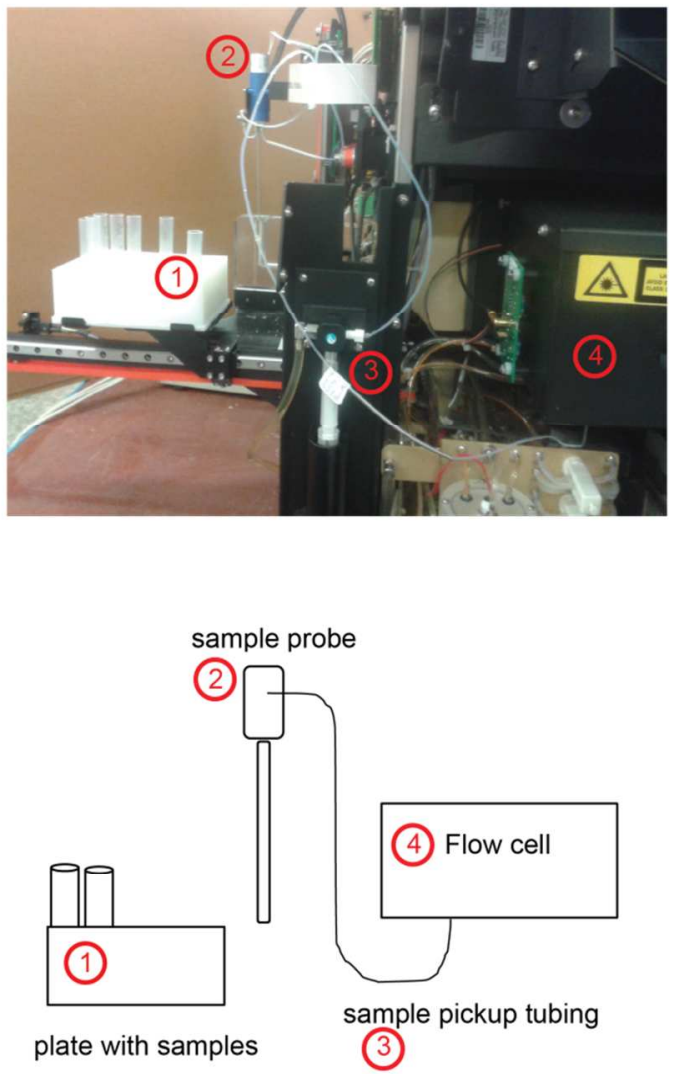

\section{B Modified sample flow in FC \\ with continous flow on-line bioassay}
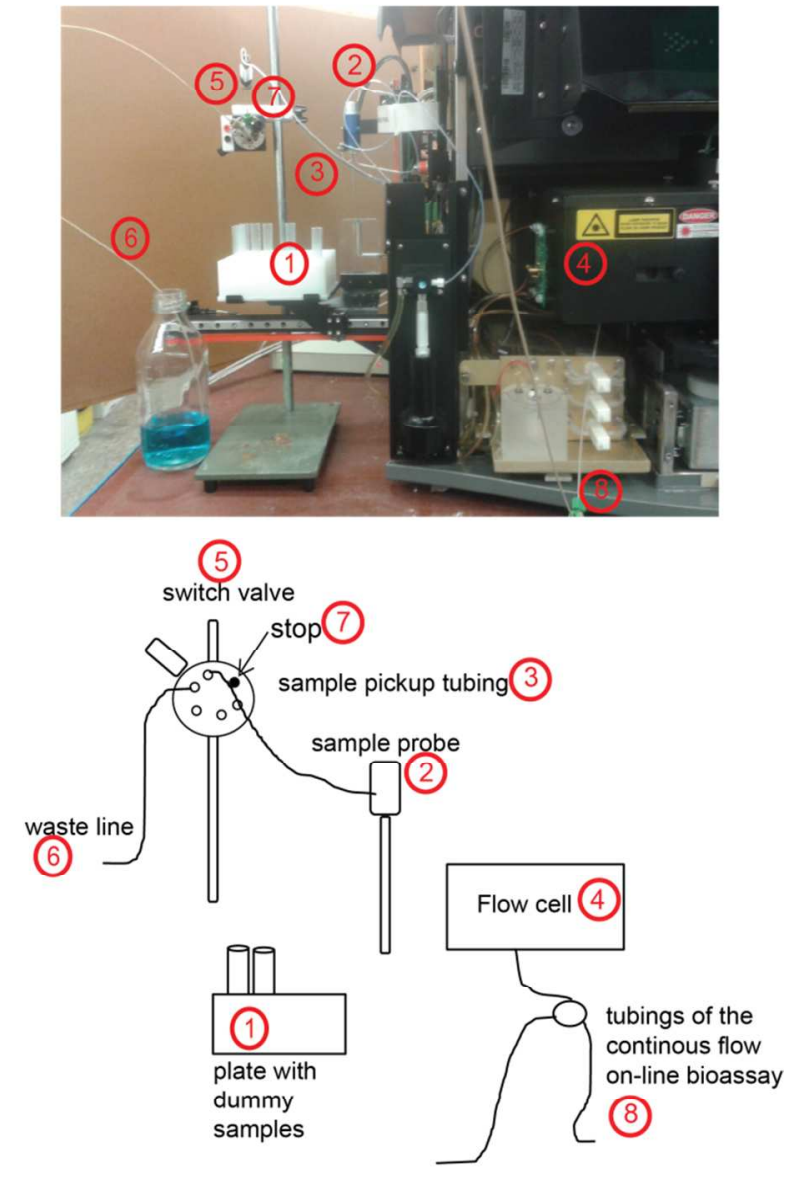
Figure S-3. Effect of using different gating during FC data acquisition. (A) shows the forward scatter (FS) $\underline{\underline{v}}$ side scatter (SS) during a regular measurement. Most of the cell population belongs to the viable SH-SY5Y cells (red circle), however fragmented (green circle) and aggregated cells (above the limit of FS) are also present in the mixture. (B) shows Time $\underline{v s}$ FL1 without using gating. The peak of a positive control is observed, however, the noise is much higher. (C) Gating as used during all data acquisition in this study. The cells outside this defined gating region are fragmented, aggregated or dead cells (aggregated cells are above the FS detection limit, fragmented cells are smaller than the normal cells in the FS detector and are also more granulated, and cells that are smaller than the SH-SY5Y cells and not showing fluorescence are also fragmented cells). Gating on the functionally responding cell population reduces the noise. (D) FL1 by time, gating on the fragmented cells. These cells are not showing a calcium response.

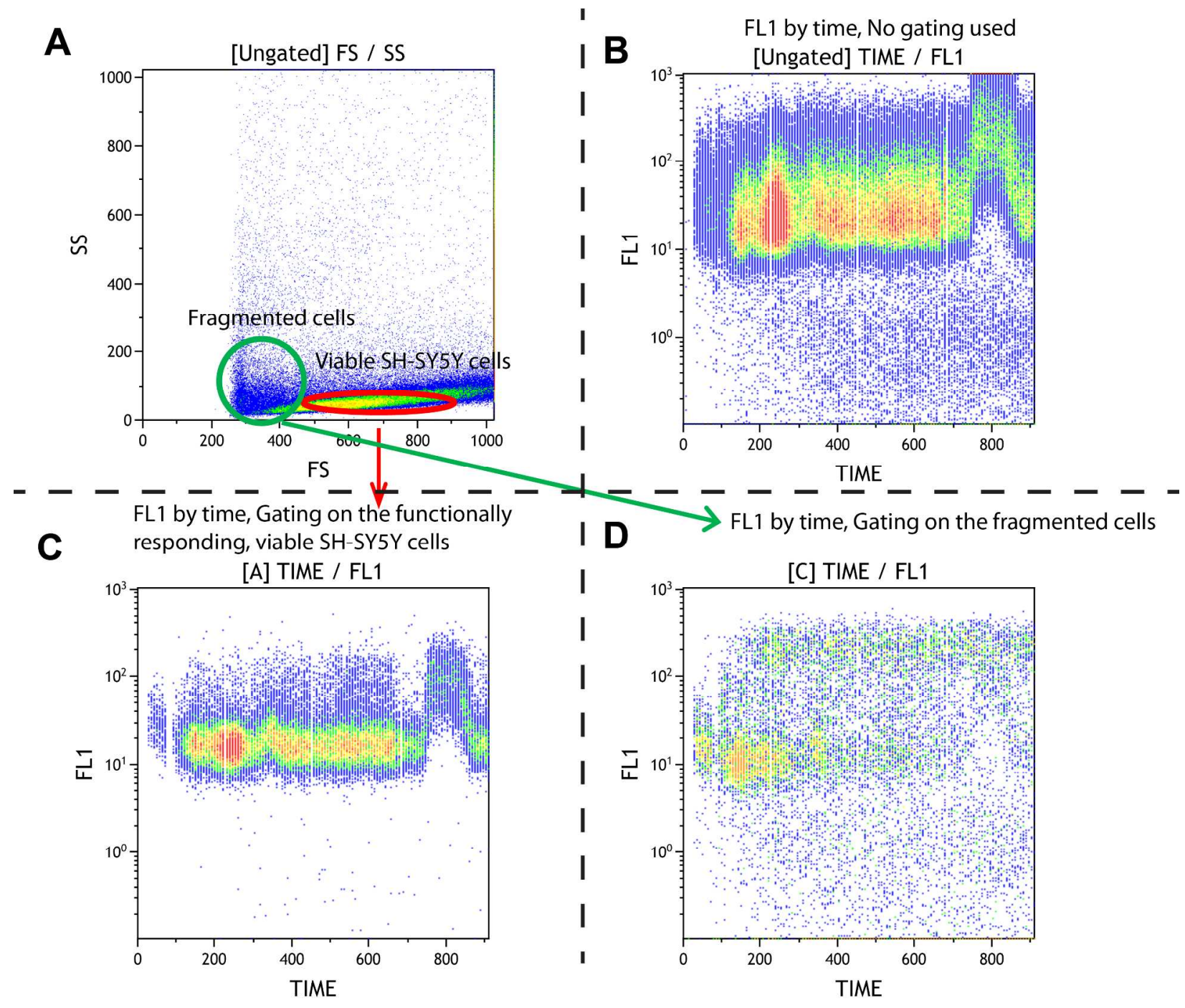


Figure S-4. Testing the effect of organic solvents and salts on the SH-SY5Y cells in plate-reader format. Various concentrations of (A) $\mathrm{ACN}$, (B) $\mathrm{MeOH}$, (C) $\mathrm{NaCl}$, (D) $\mathrm{NH}_{4} \mathrm{HCO}_{3}$ were added before the addition of $100 \mu \mathrm{M}$ of acetylcholine (ACh). Maximum tolerable concentration was defined as a decrease in the assay window of the Ca-flux signal for ACh of more than 5 percent as compared to the same experiment without the addition of the organic solvent or salt. For ACN the maximum tolerable concentration was found to be $2 \%$, for $\mathrm{MeOH} 4 \%$, for $\mathrm{NaCl} 100 \mathrm{mM}$ and for $\mathrm{NH}_{4} \mathrm{HCO}_{3} 33 \mathrm{mM}$.

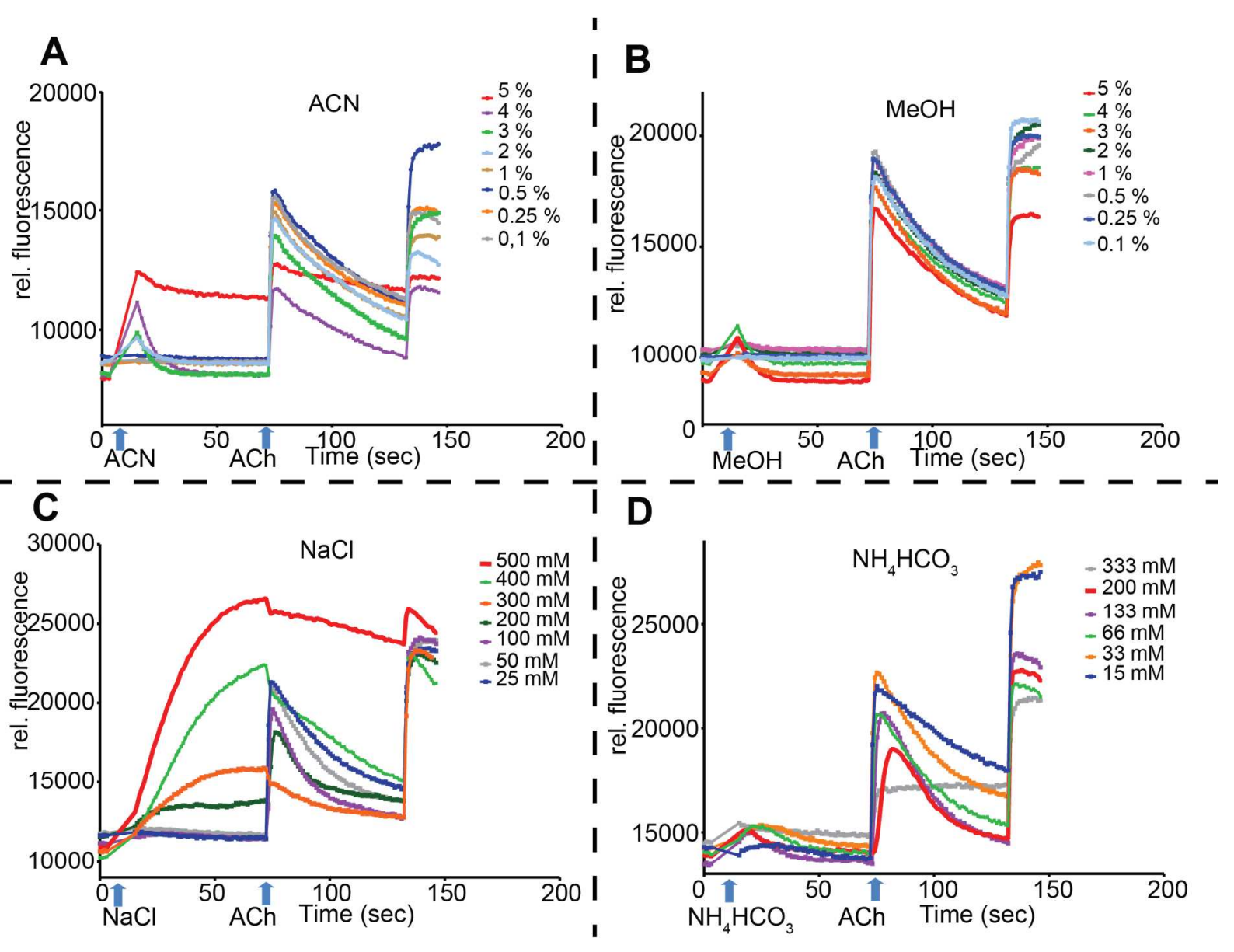


Figure S-5. Testing the effect of organic solvents on the SH-SY5Y cells in the on-line FC-LC assay format. (a) Duplicate injection of $7.45 \mu \mathrm{M}$ nicotine (final assay conc.) was tested with isocratic $0 \%, 70 \%$ (3.4\% final assay conc.), and 100\% (4.6\% final assay conc.) eluent B. The results were in correlation with the $4 \%$ max eluent conc. determined using plate reader experiments (Figure S-4), since the bioactivity peak height significantly decreased with $100 \%$ eluent $\mathrm{B}$, while the bioactivity signal was repeatable (and similar to using $0 \%$ eluent $\mathrm{B}$ ) with using 70\% eluent B. (b) and (c) Effect of a 15 min $0-70 \%$ eluent B HPLC gradient on the FC signal and on the cells in agonist mode (b) and in mixed antagonist-agonist mode (c). On Figure $\mathrm{C}$ the HPLC gradient starts after reaching the increased baseline by the continuous agonist infusion. The HPLC gradient did not cause any significant change on the baseline of the assay.
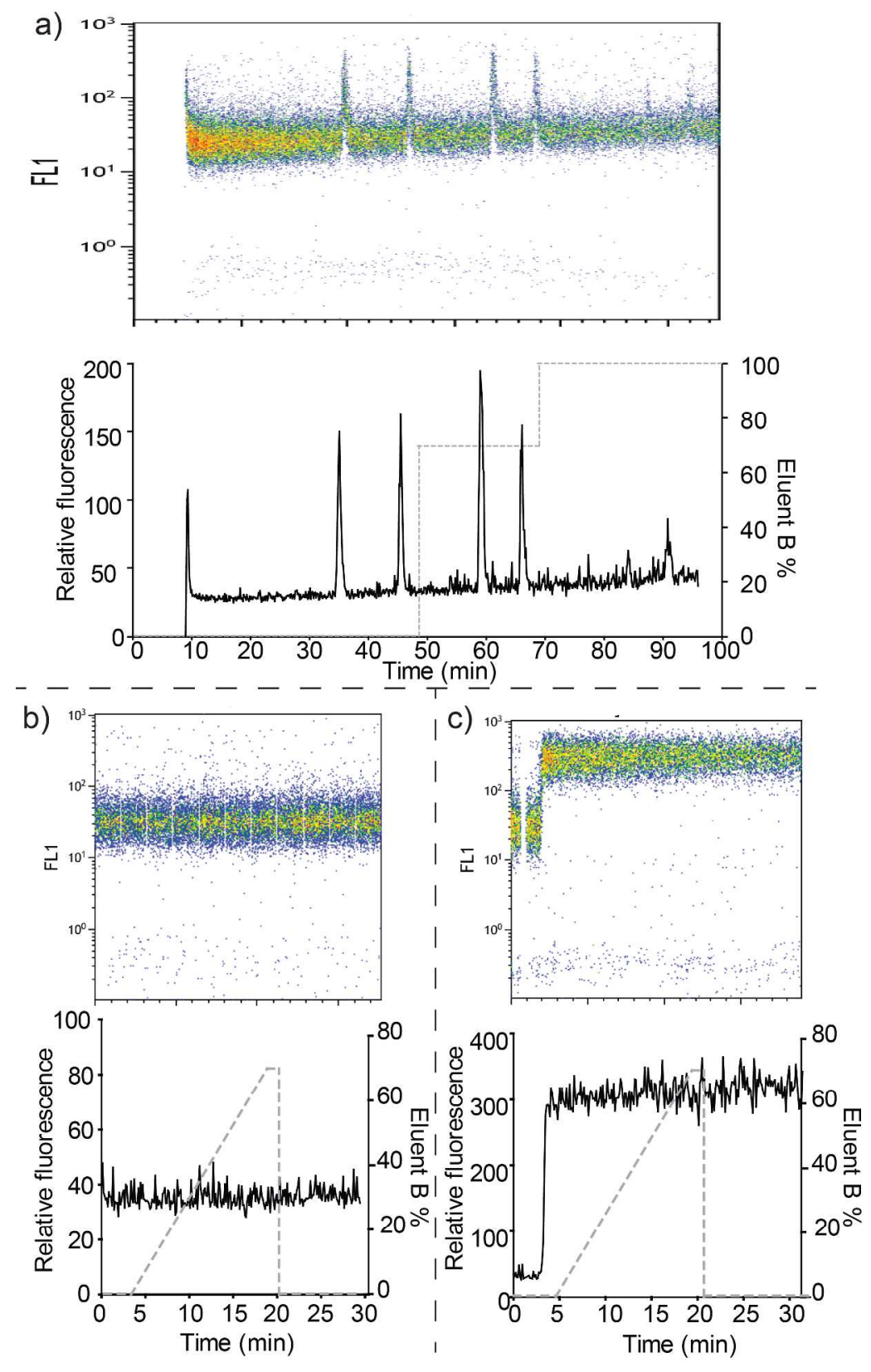
Figure S-6. Determination of cell viability using propidium iodide (PI) staining. PI is a fluorescent dye staining only dead and apoptotic cells by binding of PI to DNA and RNA which causes fluorescence enhancement with the excitation/emission maxima of $533 / 617 \mathrm{~nm}$, therefore it is excitable by the argon blue laser $(488 \mathrm{~nm})$ and the emitted fluorescence can be detected by the FL3 channel $(620 \mathrm{~nm})$ of the FC. The dye is membrane impermeable, so viable cells are not stained by PI. The cell viability assessment is demonstrated with a blank 0-70\% eluent B HPLC gradient. (a) Ungated density plot of forward scatter (FS) per side scatter (SS); the third dimension (color) represents the number of cells. Based on the size and granularity of the cells, groups of cells (gate A, C, D, and E) can be separately gated. (b, c, d, e) Figures show histogram plots of gated cells in the FL3 channel (dead cells show fluorescence): gate A, fragmented cells (b), gate C, apoptotic cells stained with PI (c), gate D, aggregated cells (d), gate E, viable, functionally responding cells (e). These histogram plots display the fluorescence intensity in the FL3 channel on the $x$-axis and the number of events (cell count) on the y-axis. Figure (e) shows that when gating on the cell population under gate E, the cells are not stained with PI, demonstrating that they are viable. Therefore during every experiment in this study gate $\mathrm{E}$ was used to select the viable cells for plotting the FC bioassay data. In (f) the percentage of viable cells (Gate E/Ungated, number of viable cells/all cells) is displayed in time. There is a very minor decrease in viable cells, however the HPLC gradient did not affect the number viable cells in the FC assay.
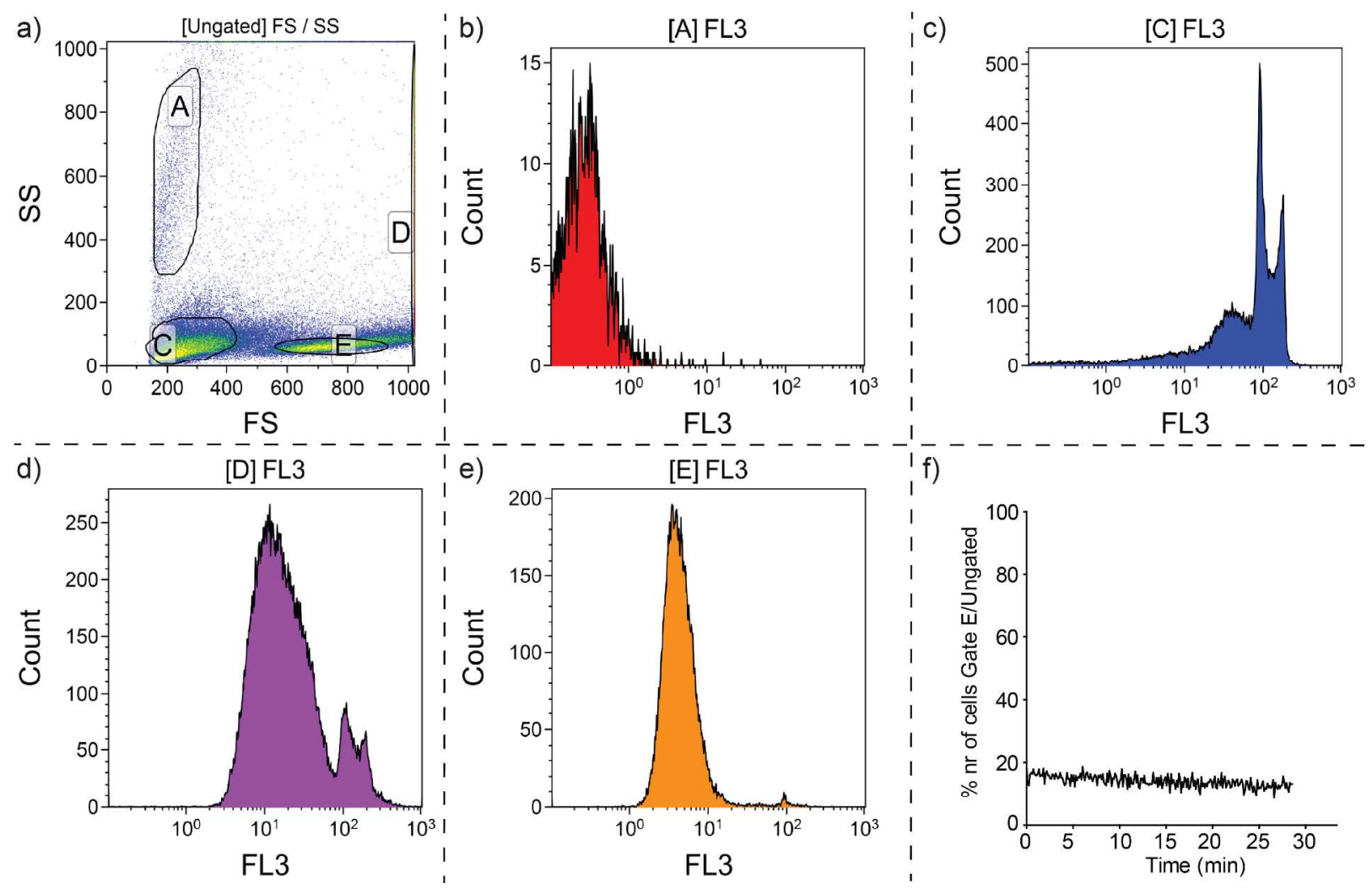
Figure S-7. Intraday repeatability and interday reproducibility in agonist mode. Repeated injections $(\mathrm{n}=9)$ of $0.746 \mu \mathrm{M}$ (injected concentration $\mathrm{c}_{\mathrm{i}}, 100 \mu \mathrm{M}$ ) PNU282987. Experiments performed on two different days are shown. The raw (a) and the converted (b) FC bioassay chromatograms are displayed. The signal window is decreasing during the measurement, however even after 1.5 hours a bioactivity peak can be still clearly distinguished from the baseline noise.

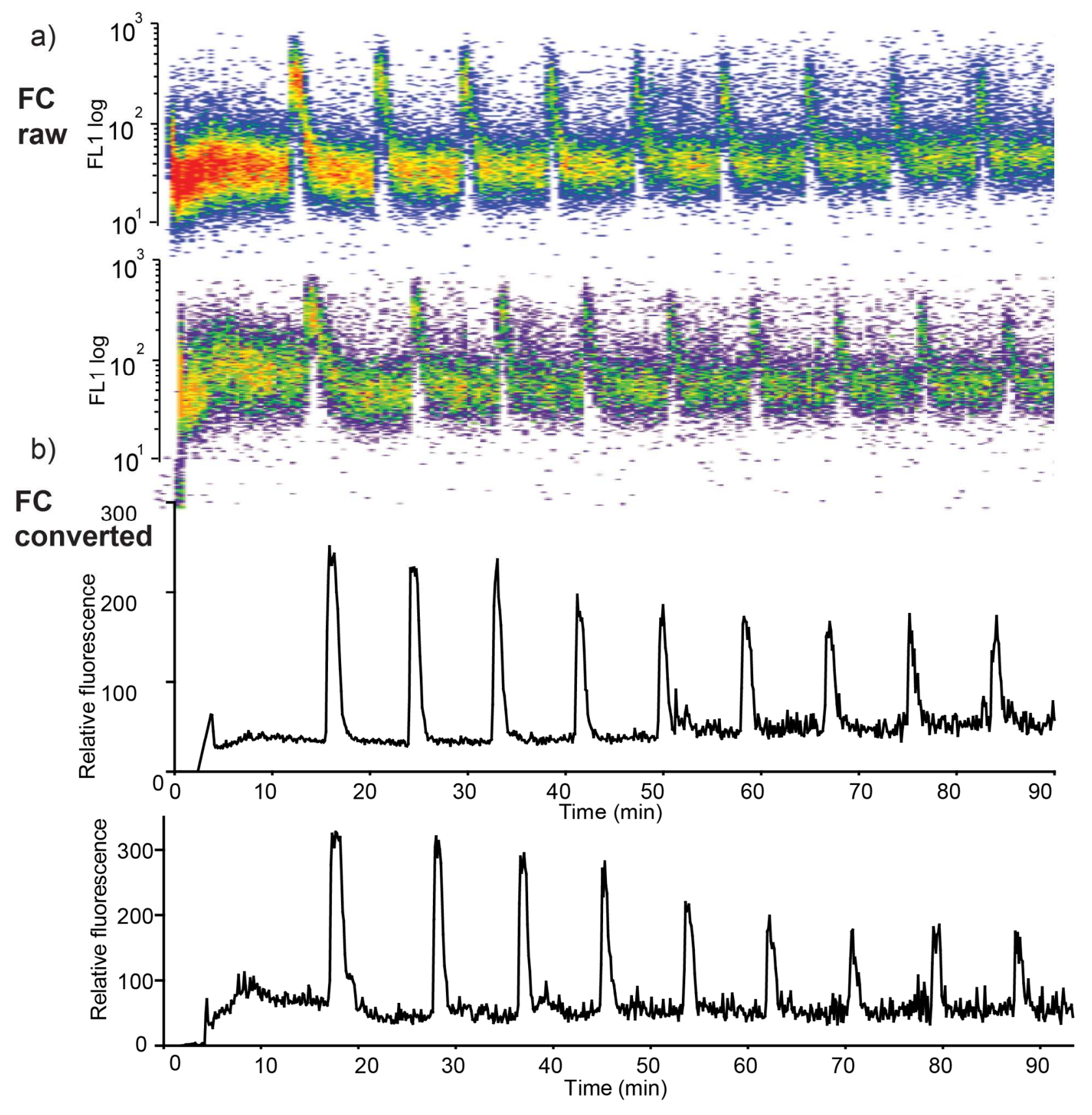


Figure S-8. Intraday repeatability and interday reproducibility in mixed antagonist-agonist assay mode. Repeated injections ( $\mathrm{n}=9$ ) of $0.1 \mu \mathrm{M}$ (injected concentration $\mathrm{c}_{\mathrm{i}}, 20 \mu \mathrm{M}$ ) MLA. Experiments performed on two different days are shown. The raw (a) and the converted (b) FC bioassay chromatograms are displayed.

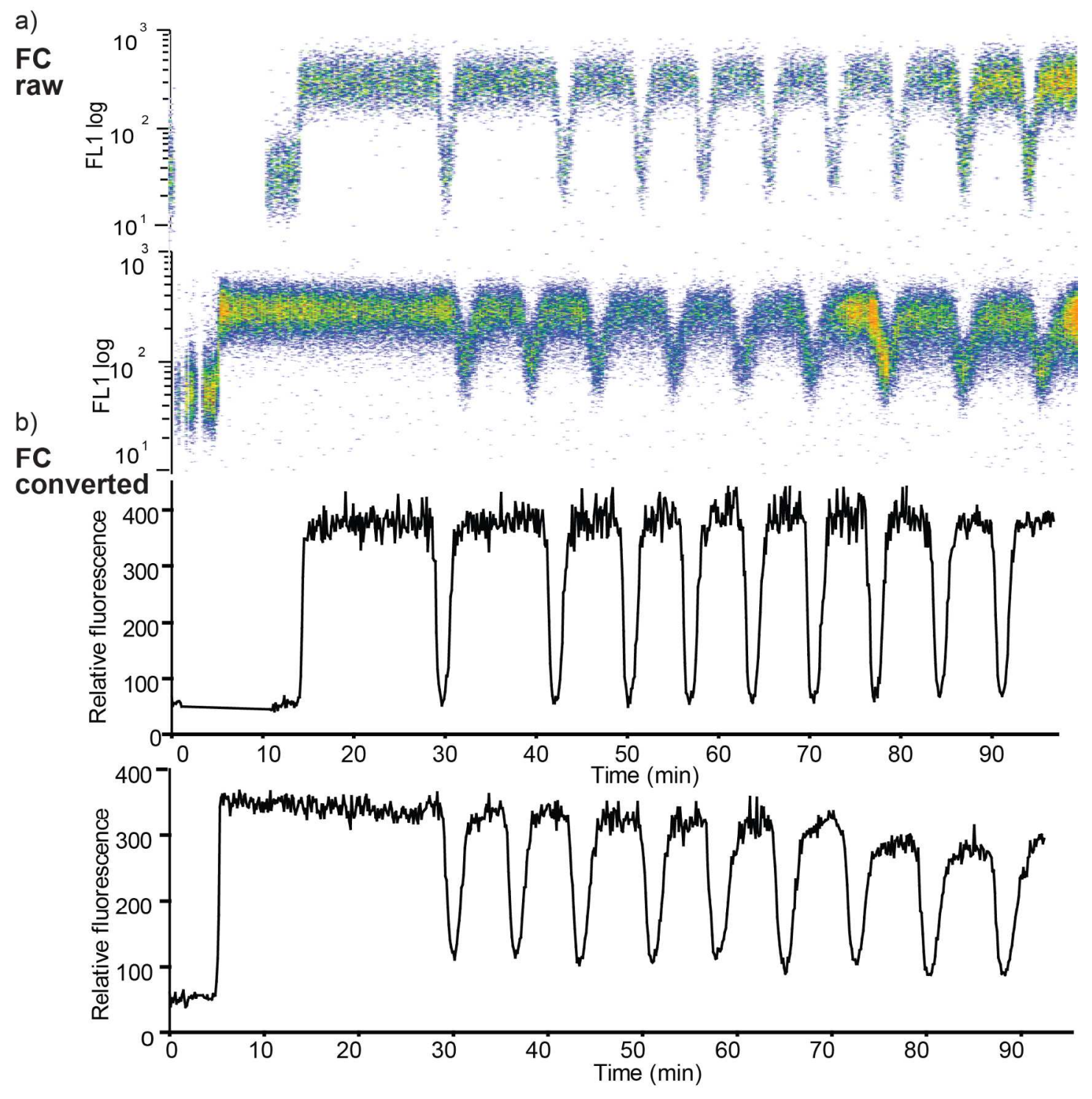


Figure S-9: Proof-of-principle experiment showing the functioning of the mixed agonistantagonist setup. The raw (a) and the converted (b) FC bioassay chromatograms are displayed in the figure. At time $0 \mathrm{~min}$ the flow of continuous nicotine was not connected. At $13 \mathrm{~min}$, nicotine ( $1 \mathrm{mM}$ injected concentration, $7.45 \mu \mathrm{M}$ final assay concentration) was injected as positive control. From $30 \mathrm{~min}$, nicotine ( $1 \mathrm{mM}$ injected concentration, $7.45 \mu \mathrm{M}$ final assay concentration) is continuously infused, which results in the permanent activation of the cells and a constant increased fluorescence signal. When an antagonist eluted from the LC column (70 and $88 \mathrm{~min}$, $50 \mu \mathrm{M}$ of MLA was injected) it temporarily blocked the continuous activation of the receptor resulting in a negative peak in the FC bioassay. When the continuous infusion of nicotine was stopped, the baseline returned to the original fluorescence level as in the beginning of the experiment without continuous nicotine infusion.

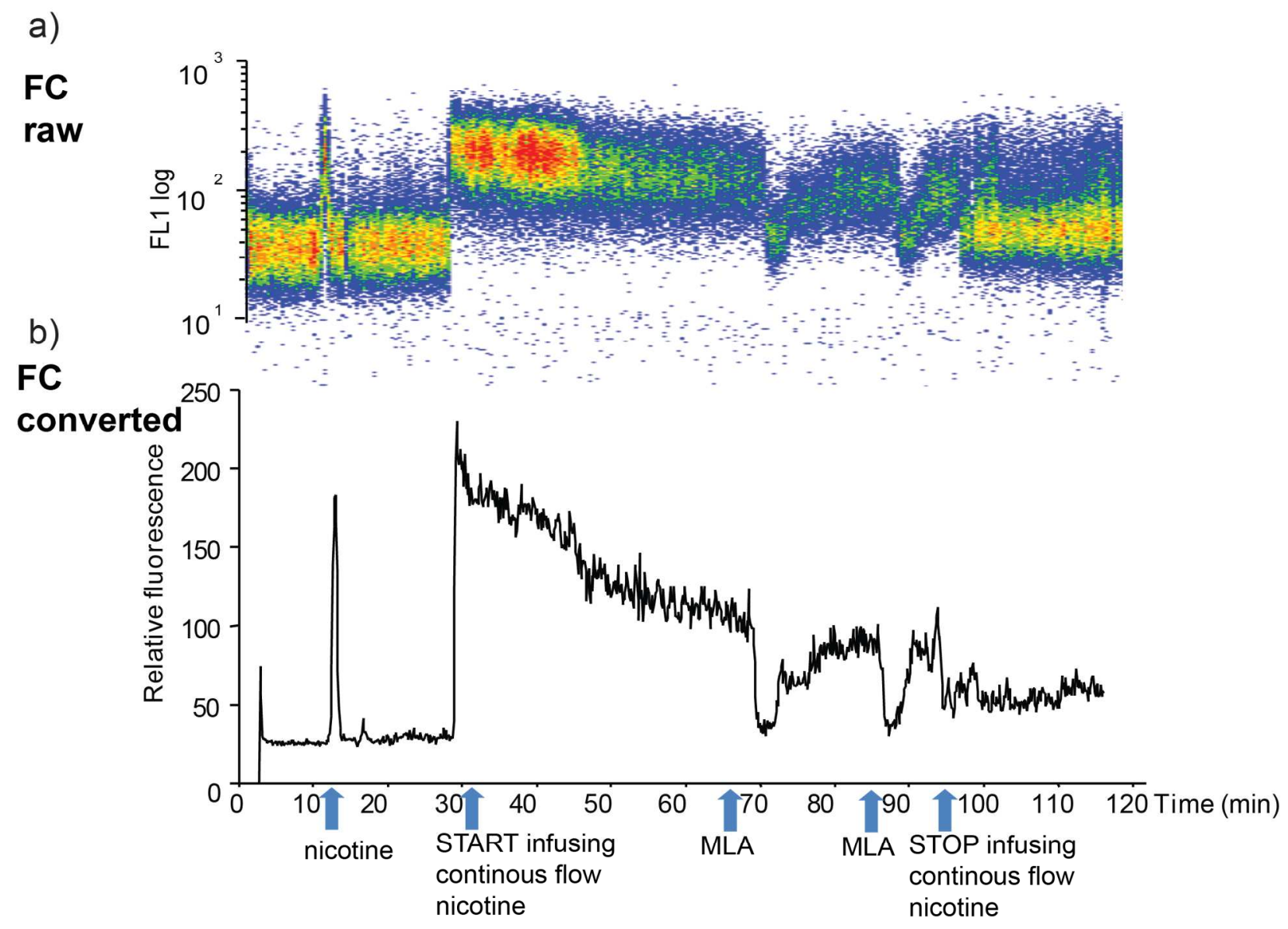


Figure S-10: Parallel agonist-antagonist measurement in antagonist mode. Gradient LC separation of nicotine and MLA. The raw (a) and the converted (b) FC bioassay chromatograms are displayed.

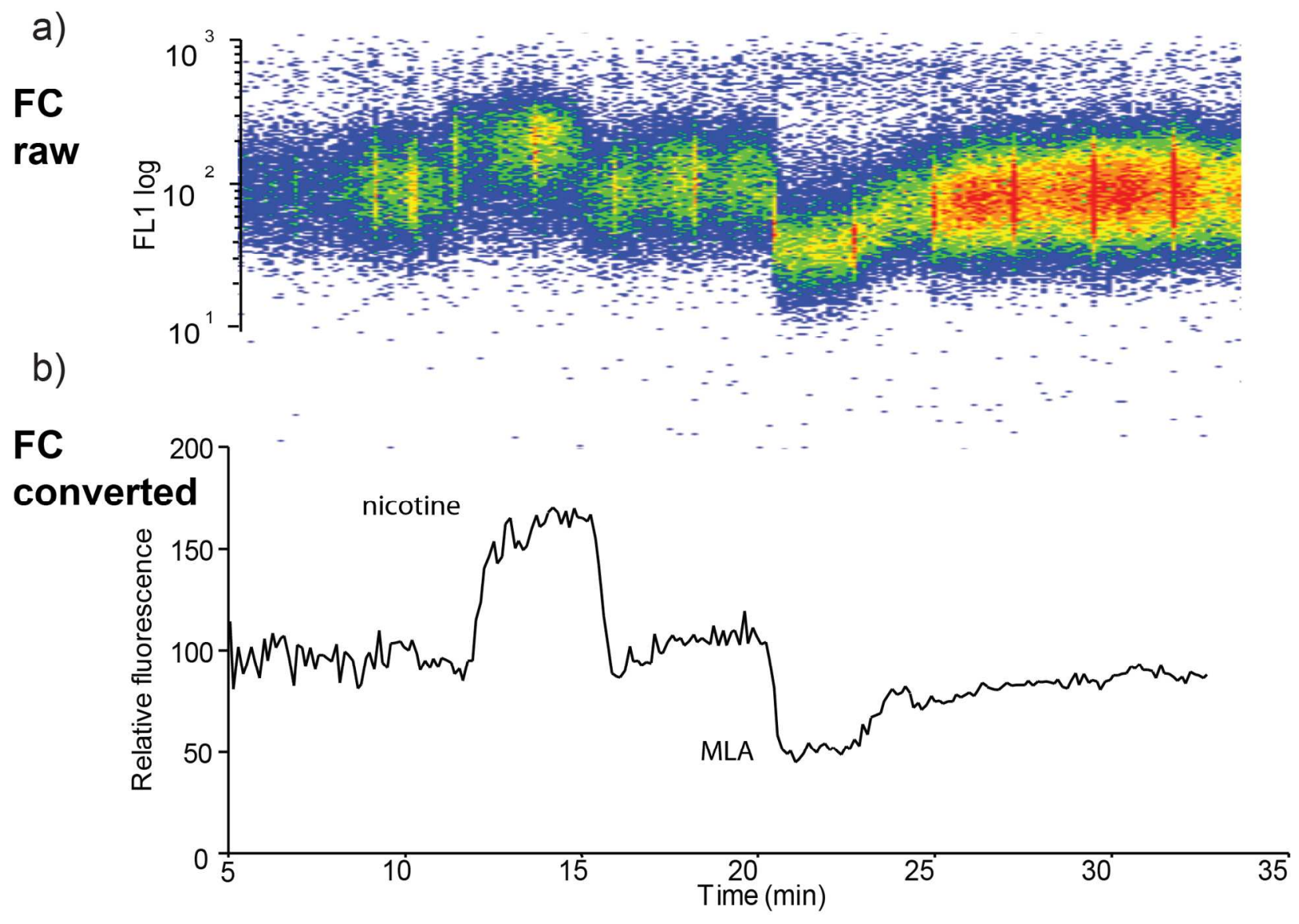


Figure S-11. On-line cell-based bioactivity screening of the Dendroapsis polylepis snake venom. The raw FC (a), the converted FC (b), and the MS (c) chromatograms are displayed. The positive peak in the bioactivity chromatograms was correlated to an ion with an $m / z$ value of 146.118 , which corresponds to acetylcholine. The negative bioactive peak corresponds to an $m / z$ value of 1142.530 and 999.845 ( +7 and +8 charged ions, respectively).

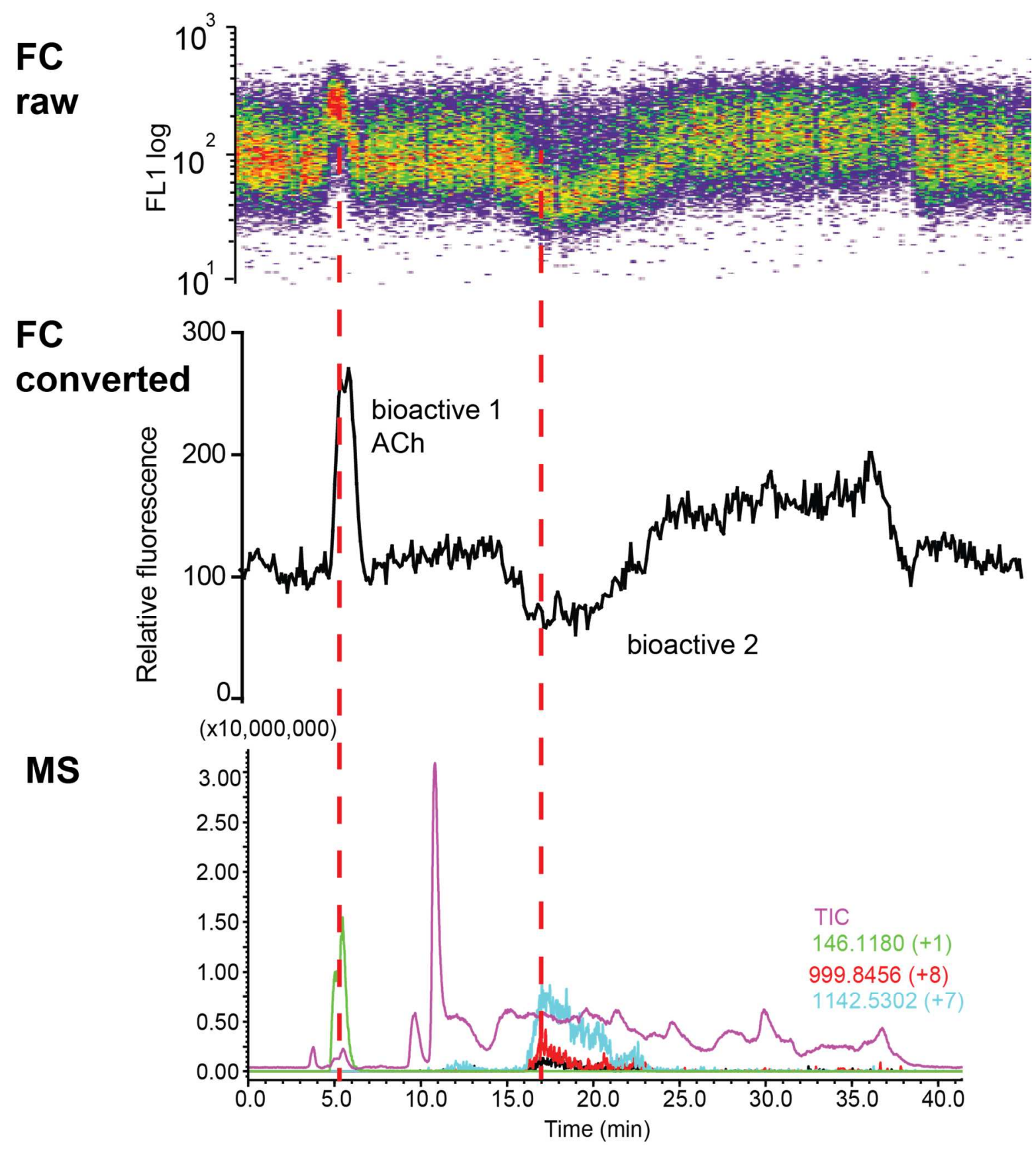

